\title{
I PREFISSI VERBALI DAL LATINO CLASSICO ALL'ITALIANO: PROBLEMI DI CLASSIFICAZIONE SU BASE ETIMOLOGICA
}

\author{
IL PREFISSO $A D$ -
}

\begin{abstract}
Summary: I examine verbal prefixation analyzing the functional changes of the Latin ad-prefix from Classical Latin to Italian. In order to conduct the research properly I needed to separate the verbs in etymological groups directly derived from Latin (Classical, Vulgar or Late Latin) from the verbs created in the Romance period and the Latin loan verbs. The different origin of the verbs influences our expectation regarding the analyzability of a given verb (the recognisability of the prefix as an independent element and its semantic value - which can be different from that of its Latin origin). This division is not as clear cut as it seems to be, because, in the case of the Italian, phonetic evidence in favour of one group or another is often missing. I present the possible solutions I found for the grouping problems using semantic evidence, comparison with the other Romance languages, dating of the given verb, etc. Furthermore, I highlight the general and specific factors which determine the assignment of a certain verb to a certain group in order to obtain a precise but still flexible set of verbal categories.
\end{abstract}

Key words: Latin and Italian, semantics, verbal prefixation, etymological grouping

\section{INTRODUZIONE}

Questo contributo fa parte di una mia ricerca sulla prefissazione verbale nel latino classico e nell'italiano, più specificamente sul cambio di funzione dei prefissi $a d$ - ed ex- nel passaggio dal latino all'italiano. Ho scelto questi due prefissi per avere degli elementi morfologici che si dimostrano produttivi nella lingua latina e continuano ad esistere ed essere produttivi anche nella lingua italiana. L'analisi del prefisso ex- nel passaggio dal latino all'italiano è stata l'argomento della mia tesi di laurea in Lingua e Letteratura Italiana, ${ }^{1}$ su cui si è basata la mia relazione al congresso internazionale

${ }^{1}$ JEKL, Á.: La prefissazione dei verbi nel latino classico e nell'italiano. Il prefisso ex- [tesi di laurea]. Budapest (Università degli Studi Eötvös Loránd) 2008. 
Formal Linguistics and the Teaching of Latin (Venezia,18-20.11.2010), poi pubblicata negli Atti. ${ }^{2}$ In questo contributo mi concentrerò sul prefisso $a d-$.

Gli sviluppi intervenuti nella mia ricerca negli ultimi anni riguardano soprattutto il metodo di analisi dei verbi prefissati, la costituzione del corpus da analizzare e una sua migliore organizzazione. Nel presente articolo vorrei quindi riprendere queste problematiche. Al punto 2 descriverò il processo di costituzione del corpus, al punto 3 presenterò la classificazione dei verbi prefissati, al punto 4 esporrò e analizzerò le problematiche emerse durante il raggruppamento dei verbi e presenterò le soluzioni possibili, al punto 5 presenterò alcuni fenomeni riscontrati nei verbi ricostruiti per il latino volgare e periodi successivi e infine al punto 6 trarrò le conclusioni finali.

\section{IL CORPUS}

Alla base del corpus dei verbi prefissati sta il $D E L I^{3}$ di cui ho raccolto tutti i verbi prefissati con $a d$-. Questo corpus è stato poi ricontrollato e rivisto in base al GRADIT Etimologico. ${ }^{4}$ Ho usato anche il Lessico di frequenza della lingua italiana contempora$n e a^{5}$ e sto integrando i dati in base al Lessico di frequenza dell'italiano parlato ${ }^{6}$ per fornire un saggio dei verbi più frequenti tra quelli prefissati con il prefisso italiano $a$ - limitazione imposta dall'enorme quantità di questi verbi presente nel lessico italiano. Come dizionari supplementari ho usato il Dictionnaire étymologique de la langue latine, ${ }^{7}$ il Thesaurus Linguae Latinae, ${ }^{8}$ il REW, ${ }^{9}$ il Battaglia,${ }^{10}$ lo Zingarelli minore, ${ }^{11}$ il TLIO ${ }^{12}$ il vocabolario Treccani,${ }^{13}$ il $D u$ Cange ${ }^{14}$ e, quando era necessario, i dizionari etimologici di altre lingue neolatine (soprattutto quello francese: $F E W^{15}$ ). Il $D E L I$

${ }^{2}$ JEKL, Á.: Verbal Prefixation in Classical Latin and in Italian. The Prefix ex-. In ONIGA, R. IOVINO, R. - GiUSti G. (eds): Formal Linguistics and Teaching of Latin. Theoretical and Applied Perspectives in Comparative Grammar. Cambridge 2011, 201-214. $2012^{2}$

${ }^{3}$ Cortelazzo, M. - Zolli, P.: DELI, Dizionario etimologico della lingua italiana. Bologna

${ }^{4}$ De Mauro, T. - MANCINI, M.: I Grandi Dizionari, Etimologico [GRADIT Etimologico]. Milano 2000.

${ }^{5}$ Bortolini, U. - TAgliavini, C. - ZAmpolli, A.: Lessico di frequenza della lingua italiana contemporanea. Milano 1972.

${ }^{6}$ DE MAURO, T. - MANCINI F.: Lessico di frequenza dell'italiano parlato. Milano 1993.

${ }^{7}$ Ernout, A. - Meillet, A.: Dictionnaire étymologique de la langue latine. Paris $1959^{4}$ (rist. 1967).

${ }^{8}$ Thesaurus Linguae Latinae. Lipsiae 1900-.

${ }^{9}$ MEYER-LÜBKE, W.: Romanisches Etymologisches Wörterbuch $[R E W]$. Heidelberg $1935^{3}$.

${ }^{10}$ BAtTAgliA, S.: Grande Dizionario della Lingua italiana. Torino 1961-2002.

${ }^{11}$ Zingarelli, N.: Zingarelli minore, Vocabolario della lingua italiana. Bologna $1994^{12}$.

12 Beltrami, P. G. - LeONARDi, L. - SQuillacioti, P.: Tesoro della Lingua Italiana delle Origi$n i[T L I O] 1997$ online: http://tlio.ovi.cnr.it/TLIO/

${ }_{13}^{13}$ Vocabolario Treccani online: http://www.treccani.it/vocabolario/

${ }^{14}$ DU CANGE, C. - DU FRESNE, CHARLES ET AL.: Glossarium medice et infimce latinitatis. Niort 1883-1887, online: http://ducange.enc.sorbonne.fr/

${ }^{15}$ WARTBURG, W. VON: Französisches Etymologisches Wörterbuch [FEW]. Bonn-HeidelbergLeipzig-Berlin-Basel 1922-2002. online: http://www.atilf.fr/FEW/ 
è stato un buon punto di partenza in quanto si basa sulle parole (titoli di lemmi) incluse nello Zingarelli minore, vocaboli usati nel linguaggio quotidiano. ${ }^{16}$ Le etimologie contenute nel DELI sono dettagliate e le parole hanno una datazione - fattori che sono di grande aiuto per l'inserimento dei singoli verbi nei gruppi etimologici. Il GRADIT Etimologico contiene un repertorio più vasto (inclusi termini tecnici di settori specifici), ed è molto utile dal punto di vista della datazione, sempre presente e più precisa, e per il fatto che indica all'inizio dei lemmi se si tratta di un 'termine tecnico' oppure di un 'regionalismo' o 'dialettalismo', oppure di un verbo semplice. Nei casi in cui l'etimologia e la datazione non bastavano per l'inserimento adeguato di un verbo in un certo gruppo etimologico, ho usato il $R E W$ per controllare se il dato verbo poteva avere un antenato romanzo oppure ho usato i dizionari etimologici delle singole lingue romanze per controllare se il dato verbo esiste anche in un'altra lingua neolatina. Il Battaglia e il vocabolario Treccani sono stati di aiuto per verificare gli arcaismi. Ovviamente le ipotesi etimologiche dei diversi dizionari elencati sopra non sempre coincidevano, e in quei casi si è dovuta prendere una decisione a favore dell'etimologia più probabile o meglio sostenibile.

\section{LA CLASSIFICAZIONE}

\subsection{Preliminari alla classificazione dei verbi}

La classificazione si basa sull'etimologia dei singoli verbi. È nata con lo scopo di rendere più facile la futura analisi dei verbi. Essa tende ad essere lineare nel tempo partendo dai verbi derivati dal latino classico per arrivare ai verbi di origine italiana. $\mathrm{Ci}$ sono tuttavia gruppi etimologici per cui non può essere stabilita una sequenza cronologica, ma che sono contemporanei e si differenziano solo per il modo in cui sono attestati o ricostruiti - parlerò di questi gruppi più in dettaglio fra poco. In base alla loro etimologia possiamo dividere i verbi prefissati in tre grandi gruppi etimologici: a) verbi di discendenza diretta di origine latina, b) verbi di discendenza indiretta di origine latina dotta oppure verbi mutuati da altre lingue e da dialetti italiani e infine c) verbi di creazione italiana. Nella futura analisi semantica dovranno poi essere trattati separatamente i verbi creati con metodi di derivazione marcati dal punto di vista del rapporto semantico tra il prefisso e il verbo.

\subsection{Metodi di derivazione marcati dal punto di vista dell'analizzabilità del rapporto semantico tra il prefisso e il verbo}

Come accennato precedentemente, ci sono alcuni metodi di derivazione che sono marcati ${ }^{17}$ dal punto di vista dell'analizzabilità del rapporto semantico tra il prefisso e il

\footnotetext{
${ }^{16} \operatorname{DELI}$ (n. 3) 4-5.

${ }^{17}$ In quanto rappresentano delle deviazioni rispetto al tipo base.
} 
verbo. Sono metodi che hanno un'influenza decisiva - o comunque importante - sulla semantica del verbo prefissato. Nel caso dei verbi modificati tramite cambio di prefisso questa influenza è concreta e positiva. Per i parlanti della data epoca il prefisso sostituito non esprimeva più quel significato che invece sentivano vivo nel prefisso ad-: così, per mantenere la trasparenza del verbo prefissato, hanno modificato il suo prefisso (per es. aspettare per il latino classico exspectāre). Possiamo presupporre una tendenza contraria nel caso dei verbi denominali dato che il prefisso probabilmente non esprime più un significato trasparente, ma, nella maggioranza dei casi, si dissolve nel significato del nome o in quello dell'aggettivo. In questo modo il suffisso verbale si aggiunge a un significato già 'sintetizzato' per es. assedio - assediare, e quindi il ruolo del prefisso non è più riconoscibile. Stanno in mezzo tra i verbi modificati tramite cambio di prefisso e quelli denominali i verbi modificati tramite l'incrocio di più forme. Nel caso di questi verbi proprio per interazione delle fonetiche e dei significati di più verbi non si può predire se questi verbi saranno analizzabili dal punto di vista semantico o meno e, in caso affermativo, fino a che grado può arrivare l'analisi semantica. ${ }^{18}$ Tutti i verbi creati con i metodi descritti sopra sono comunque inseriti nei diversi gruppi etimologici e la loro separazione sarà necessaria solo nella fase dell'analisi semantica dei singoli verbi.

\subsection{Classificazione dettagliata}

\begin{tabular}{|l|l|l|l|}
\hline a, & \multicolumn{3}{|l|}{ Verbi prefissati italiani derivati dal latino - discendenza diretta } \\
\hline & $1)$ & verbi attestati nel latino classico (per es. accendere) \\
\hline & $2)$ & verbi attestati nel latino tardo o ricostruiti per il latino volgare \\
\hline & & $2) 1$. & ricostruiti per il latino volgare (per es. accorciare) \\
\hline & & $2) 2$. & attestati nel latino tardo (per es. allattare) \\
\hline b, & Verbi prefissati di discendenza indiretta \\
\hline & $1)$ & prestiti dotti dal latino (per es. abbreviare) \\
\hline & $2)$ & prestiti da un'altra lingua (soprattutto romanza) \\
\hline & \multicolumn{4}{|l|}{ 2)1. } & interamente di origine straniera (per es. abbonare) \\
\hline & & 2)2. & di base straniera + prefisso latino/italiano (per es. \\
& & accantonare) \\
\hline & $3)$ & prestiti da dialetti italiani (per es. abbuffarsi) \\
\hline c, & Verbi prefissati di creazione italiana (prefissati con il prefisso italiano $a(d)$-) \\
\hline & $1)$ & verbi prefissati (per. es. affidare) \\
\hline & $2)$ & verbi parasintetici (per es. abbracciare) \\
\hline
\end{tabular}

Tabella 1 .

${ }^{18} \mathrm{Nel}$ gruppo dei verbi modificati tramite l'incrocio di più forme si annoverano quattro verbi. Ognuno dei quattro verbi ha come base due forme che si sono sovrapposte - per es. attorcigliare: attorcere $\sim$ attortigliare, annusare: muso annasare. Dato che nel caso di ciascuno dei quattro verbi una delle forme base non esiste più oppure non è più in uso nell'italiano contemporaneo, il confronto delle forme base non è più realizzabile. 
La Tabella 1 rappresenta in maniera sintetica la classificazione e la sottoclassificazione dei gruppi etimologici che sta alla base della mia ricerca.

Nel gruppo a, troviamo

1) i verbi derivati dal latino classico e

2) i verbi ricostruiti per il latino volgare o attestati nel latino tardo.

Nel gruppo b, troviamo

1) i verbi di origine latina dotta,

2) i verbi mutuati da altre lingue (soprattutto romanze) e

3) verbi mutuati da dialetti italiani.

Infine, nel gruppo c, troviamo

1) $i$ verbi prefissati e

2) i verbi parasintetici.

I verbi di discendenza diretta dal latino hanno subito i cambiamenti fonologici, morfologici e semantici avvenuti durante lo sviluppo della lingua italiana a partire dal latino, mentre i verbi di discendenza indiretta dal latino (verbi dotti) non hanno subito questi cambiamenti (fatta eccezione per la semplificazione o modificazione dei gruppi consonantici non ammessi nell'italiano, per es. adattare e non *adaptare). Nel caso dei verbi presi in prestito da altre lingue invece tutti i tipi di cambiamenti linguistici sono possibili.

La denominazione dei gruppi etimologici segnala l'inizio dell'attestazione per il verbo in questione, quindi un verbo inserito nel gruppo dei verbi derivati dal latino classico è attestato dal periodo classico in poi, mentre un verbo inserito nel gruppo dei verbi derivati dal latino tardo è attestato dal periodo tardo in poi. L'eventuale origine preclassica dei verbi latini non è stata presa in considerazione in quanto intendiamo studiare il cambiamento di significato dal latino classico fino alla lingua italiana, e non l'origine del latino stesso.

Per un ulteriore fattore etimologico si è introdotta una sottocategorizzazione all'interno dei verbi mutuati da altre lingue tenendo separati i verbi di origine straniera di forma prefissata e i verbi in cui solo la base è di origine straniera, mentre il prefisso è il latino/italiano $a d$-. Il motivo di questa separazione è simile a quello descritto nel paragrafo precedente per i verbi modificati tramite cambio di prefisso: l'aggiunta del prefisso $a d$ - conferma la produttività di questo prefisso.

\subsection{Parallelismo cronologico}

Questo fenomeno si riscontra nel caso dei verbi ricostruiti per il latino volgare, che devono essere considerati contemporanei ai verbi attestati nel latino tardo, poi nel caso dei verbi derivati dal latino medievale, contemporanei ai verbi di creazione italiana. Nel primo caso si tratta semplicemente del fatto che una parte dei verbi è attestata nel latino tardo, mentre un'altra parte no. La ricostruzione dei verbi di 'derivazione popo- 
lare' è tuttavia assicurata dall'esistenza di quei verbi nelle lingue romanze. Un problema supplementare è dato dal fatto che il concetto di latino tardo utilizzato nelle classificazioni dei dizionari etimologici è un concetto molto ampio e complesso i cui confini sono difficilmente identificabili in modo concreto sull'asse temporale. E con questo arriviamo al secondo parallelismo, alla convivenza del latino medievale con i primordi della lingua italiana. Il latino del periodo dal IX al XIII secolo non è più una fonte di discendenza lineare, ma una lingua parallela all'italiano da cui si prendono i prestiti. Ovviamente conta il registro della parola mutuata in quanto una parola di uso dotto è più probabilmente identificabile come prestito dal latino medievale che come una parola di discendenza diretta o una creazione italiana. In altri casi, invece, verbi attestati nel latino medievale probabilmente sono la trasposizione in latino di verbi formatisi nelle lingue romanze. In molti casi è dunque difficile decidere se si tratta di un prestito dal latino medievale oppure di un verbo di discendenza latina diretta o di creazione romanza che è stato assunto anche dal latino medievale. Sono quindi molte le vie tramite cui i verbi latini possono arrivare all'italiano e si deve sempre prendere una decisione specifica, caso per caso, prendendo in considerazione diversi fattori come la datazione, il registro, il significato oppure la fonetica-morfologia della data parola (nel caso quando è di aiuto) - come sarà dimostrato anche negli esempi concreti del punto 4 .

\section{ANALISI DEI PROBLEMI}

Durante il raggruppamento dei verbi ho affrontato diversi problemi le cui soluzioni si differenziano a seconda delle caratteristiche del verbo stesso. Tramite campioni scelti cercherò di presentare i fattori che sono risultati decisivi per la classificazione dei relativi verbi.

\subsection{Fattore fonetico/morfologico}

Nel caso del verbo abbassare e dei verbi annichilare/annichilire è stata la fonetica il fattore decisivo per fissare la collocazione dei relativi verbi nei gruppi etimologici sopracitati. 1) Il verbo abbassare secondo il DELI deriva dal latino parlato *adbassiāre, derivato di bassu(m) 'basso'. ${ }^{19}$ Il GRADIT Etimologico lo riporta come un derivato parasintetico di basso attestato dalla fine del XIII sec. ${ }^{20}$ Il tratto morfologico decisivo è stato la mancanza della $-i$ - nel suffisso. Il $R E W$ non contiene una forma senza la $-i$ - per cui il verbo è stato interpretato come creazione italiana ed inserito nel gruppo dei verbi parasintetici di origine italiana. 2) I verbi annichilare/annichilire derivano - sia secondo il DELI sia secondo il GRADIT Etimologico - dal latino tardo

${ }^{19} \operatorname{DELI}$ (n. 3) 36.

${ }^{20}$ GRADIT Etimologico (n. 4) 2. 
adnihilāre. ${ }^{21}$ Tuttavia per la consonante rappresentata dal digramma -ch- (tipica della grafia medievale nichil per nihil, corrispondente alla pronuncia scolastica di $h$ latina intervocalica) siamo di fronte a verbi di origine dotta, dalla filosofia medievale. Anche il registro di uso di questi verbi conferma questa classificazione.

\subsection{Attestazione in altre lingue romanze}

Nel caso dei verbi accordare, allentare e atterrire sono state le attestazioni in altre lingue romanze a determinare l'origine dei verbi. 1) Nel caso del verbo accordare l'etimologia è incerta. Si pensa che il verbo ha potuto avere due accezioni: una con il significato (originariamente) musicale che risale ad un latino parlato *acchordāre (verbo parasintetico creato sulla parola chorda). L'altra accezione invece risalirebbe ad un latino parlato *accordāre creato in base al verbo concordāre con cambio di prefisso. In più queste accezioni si potevano anche mescolare. ${ }^{22}$ Il $R E W$ non è di aiuto in quanto non contiene né un verbo *accordāre né un verbo *acchordāre (neanche come possibili derivati di chorda). Tuttavia questo verbo è presente anche nel francese (accorder) e nello spagnolo (acordar). In base a queste attestazioni possiamo presupporre che questi verbi hanno dovuto avere un antenato comune nel latino volgare (e quindi raggruppare il verbo italiano accordare tra i verbi ricostruiti per il latino volgare). 2) Per il verbo allentare sono state proposte due etimologie. Secondo il DELI si tratta di un verbo parasintetico derivato da lento - quindi creazione italiana - che però si basa sul verbo latino medievale allentāre (sec. IX), in più potrebbe esser stato preceduto da un latino parlato *allentāere, ${ }^{23}$ derivato da lentus. Anche il GRADIT Etimologico ipotizza la derivazione parasintetica italiana con una datazione per il $1294 .{ }^{24}$ Controllando il $R E W$ si è trovato che questo verbo è attestato in altre tre lingue neolatine e in più dialetti italiani. ${ }^{25}$ In base a questo dato il verbo è stato incluso nel gruppo dei verbi ricostruiti per il latino volgare. Infine 3) il verbo atterrire è un derivato sulla base del verbo latino terrēre. Il $R E W$ non contiene un verbo ${ }^{* *}$ atterrire per cui si potrebbe pensare ad una creazione italiana. Il problema è che il verbo base (il latino terrēre) non sopravvive nell'italiano, quindi il verbo si è dovuto creare prima dei primordi della lingua italiana. Qui viene d'aiuto la lingua spagnola che attesta il verbo aterrar, confermando l'origine latina volgare di questo verbo.

${ }^{21}$ DELI (n. 3) 206; GRADIT Etimologico (n. 4) 100-101.

${ }^{22}$ DELI (n. 3) 51; GRADIT Etimologico (n. 4) 15.

${ }^{23}$ DELI (n. 3) 84.

${ }^{24}$ GRADIT Etimologico (n. 4) 63.

${ }^{25} R E W$ (n. 9) 28; per es. il logud. allentare 'placarsi, sciogliere', l'abruzz. allendá e il marchigiano allentare 'smettere di piovere'. 


\subsection{Significato e registro di uso dei verbi}

Nel caso dei verbi affondare, aggiornare a aggiustare è stato il significato e il registro di uso dei verbi il fattore decisivo per la loro collocazione nei diversi gruppi etimologici. 1) Nel caso del verbo affondare ci sono stati due fattori decisivi per la collocazione del verbo: a, il significato e b, le attestazioni in altre lingue romanze. Secondo il GRADIT Etimologico deriva dal lat. medievale affundāre $(1252-1258){ }^{26}$ Il DELI lo considera un verbo parasintetico su fondo (quindi di creazione italiana). ${ }^{27}$ Il significato e il registro di uso del verbo non appoggiano una derivazione dotta, così il verbo non potrebbe essere un prestito dal latino medievale. Inoltre, non si tratta neanche di un verbo di creazione italiana in quanto il $R E W$ contiene un verbo *affundāre con attestazioni in altre quattro lingue neolatine (fr. ant. afonder, sp. afondar e port. afundar con il significato 'mandare a fondo, sommergere' e rum. afunda con il significato 'profondare'). Un'ulteriore fatto importante - che riguarda il significato del verbo è che il verbo affondare non si connette semanticamente con il verbo fondare (derivato a sua volta dal latino classico fundāre), ${ }^{28}$ ma con il nome fondo (derivato a sua volta dal latino classico fundus) ${ }^{29}$ In base alle considerazioni presentate sopra, il verbo è stato classificato nel gruppo dei verbi ricostruiti per il latino volgare - tenendo presente che si tratta di un verbo parasintetico nato nel latino volgare. 2) Il verbo aggiustare ha ricevuto proposte di etimologia simili a quelle per il verbo affondare in quanto potrebbe essere un verbo parasintetico italiano derivato da giusto ${ }^{30}$ oppure possibilmente anche un verbo derivato dal latino medievale adiustāre. ${ }^{31}$ Il verbo è presente anche nella lingua francese (ajuster), ma non si trova nel $R E W$. Dato che il significato e registro di uso del verbo (all'inizio usato in un senso più ristretto per pesi e sim.) sostengono più la derivazione italiana, il verbo è stato classificato nel gruppo dei verbi di origine italiana. 3) Infine il verbo aggiornare ha una semantica ed un'etimologia abbastanza complesse. Deriva dal verbo francese ajourner con il significato 'assegnare una data', attestato la prima volta nel sec. XIII. ${ }^{32}$ Questo verbo viene poi ripreso "insieme col der. aggiornamento, nel sign. di 'rinviare' (detto soprattutto di sedute parlamentari): in questa accez. le vc. si diffondono dall'Inghilterra $[. .$.$] in Francia e (spesso tramite il fr.) nelle altre lingue europee". 33$ Semanticamente il verbo è un calco (prima) sul verbo francese ajourner (poi sul verbo inglese update), e in base al significato è stato raggruppato tra i verbi prestiti da un'altra lingua.

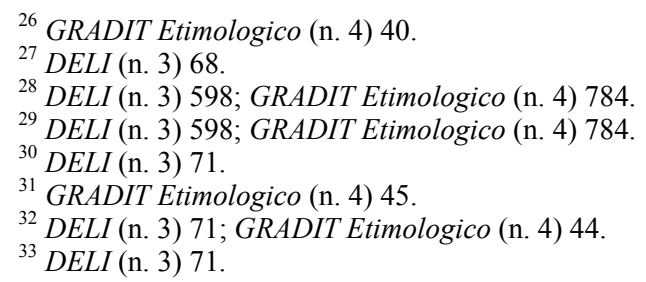




\subsection{Datazione}

Nel caso del verbo abbinare la datazione è stato uno dei fattori decisivi per la collocazione del verbo nel relativo gruppo etimologico. Abbinare è un verbo la cui parola base è di origine latina classica (binnī; sia in base a $D E L I^{34}$ sia in base a GRADIT Etimologico $^{35}$ ), ma nel latino classico non è attestato nessun verbo prefissato *abbinnāre. Per la parola base e per il significato il verbo va considerato di origine dotta, il che è confermato anche dalla sua tarda datazione (l'anno 1636 - sia in base a $D E L I^{36}$ sia in base a GRADIT Etimologico $\left.{ }^{37}\right){ }^{38}$

\section{ALCUNE PARTICOLARITÀ DEL GRUPPO DEI VERBI RICOSTRUITI PER IL LATINO VOLGARE}

Il gruppo dei verbi ricostruiti per il latino volgare ha presentato il maggior numero di difficoltà dal punto di vista della classificazione. Un punto di riferimento è stato il $R E W$, dove sono stati controllati tutti i verbi prefissati con ad-attribuiti al latino volgare dai dizionari etimologici usati. Nel caso in cui non erano presenti nel $R E W$ si è fatto ricorso alle informazioni e ai criteri sopra descritti per stabilire la derivazione di ciascun verbo.

Senza addentrarmi nell'analisi dettagliata dei verbi (ancora in corso), vorrei accennare a due fenomeni che risultano molto produttivi nel latino volgare e di cui uno avrà un'espansione enorme nelle lingue romanze. Il primo fenomeno è il cambio di prefisso (per es. affogare per il latino tardo offōcāre) oppure l'aggiunta di un prefisso nuovo al prefisso precedente - prefissi doppi (per es. accorgersi $[$ ad- + corrigo $[$ con$\left.\left.+r e g \bar{o}]^{39}\right]\right)$. In tutti e due $\mathrm{i}$ casi si tratta della reinterpretazione del verbo prefissato, e come è stato detto precedentemente, questo fatto è un segnale forte della produttività del prefisso $a d$-.

L'altro fenomeno che si riscontra esaminando i verbi ricostruiti per il latino volgare è la parasintesi (per es. adirarsi), cioè l'aggiunta contemporanea di un prefisso e di un suffisso alla parola base. Questi tipi di verbi esistevano già sporadicamente anche nel latino antico e nel latino classico, ma la loro diffusione, e l'affermazione della parasintesi come processo di derivazione avviene nella tarda latinità e soprattutto nel

${ }^{34} \operatorname{DELI}$ (n. 3) 37.

${ }^{35}$ GRADIT Etimologico (n. 4) 3.

${ }^{36} \operatorname{DELI}$ (n. 3) 37.

${ }^{37}$ GRADIT Etimologico (n. 4) 3.

${ }^{38}$ Il verbo abbinare si ritrova nei dialetti settentrionali con il significato 'riunire', e in quel caso siamo probabilmente di fronte a un verbo del latino volgare. Il verbo del linguaggio stantard però - sia per il suo significato sia per la sua tarda attestazione - risulta essere un verbo di origine dotta. La possibilità di un cambio di prefisso rispetto a combināre non è stata presa in considerazione in quanto il verbo combinare ha un significato diverso rispetto ad abbinare.

${ }^{39} \mathrm{Il}$ verbo prefissato corrigō era probabilmente una forma poco trasparente già nell'antichità, a causa dell'apofonia che ha oscurato la forma originaria della radice verbale. 
latino volgare..$^{40} \mathrm{E}$ avrà poi una fortuna estrema nelle lingue romanze, il che è dimostrato per la lingua italiana, e per il prefisso esaminato, dal fatto che dei 58 verbi più frequenti di creazione italiana 45 sono parasintetici.

\title{
6. CONCLUSIONE
}

Nel presente articolo è stata presentata la classificazione ottenuta per i verbi prefissati con $a d$ - da me raccolti. Sono stati poi presentati i problemi incontrati durante il raggruppamento etimologico di questi verbi e si è accennato anche ad alcuni fenomeni riscontrabili nel gruppo dei verbi ricostruiti per il latino volgare. Si è cercato di dimostrare come si possono risolvere i problemi incontrati usando il maggior numero di informazioni a disposizione, come le ipotesi etimologiche dei diversi dizionari, le attestazioni, i presupposti antenati volgari, la fonetica delle forme, i verbi paralleli reperibili in altre lingue romanze, il significato dei verbi, il registro d'uso e infine la datazione. Il raggruppamento non è chiuso, ci sono interazioni tra i diversi gruppi, e c'è spazio per ulteriori eventuali modifiche se richiesto da una futura analisi più dettagliata. Tuttavia credo di essere riuscita a trovare ragioni valide su cui basare la collocazione dei singoli verbi e in questo modo facilitare la loro analisi.

\author{
Ágnes Jek1 \\ Corso di Dottorato in Linguistica Romanza \\ Università degli Studi Eötvös Loránd \\ Budapest \\ Ungheria \\ agnes.jek1@gmail.com
}

Open Access. This is an open-access article distributed under the terms of the Creative Commons Attribution 4.0 International License (https://creativecommons.org/licenses/by/4.0), which permits unrestricted use, distribution, and reproduction in any medium, provided the original author and source are credited, a link to the CC License is provided, and changes - if any - are indicated. (SID_1)

${ }^{40}$ Cfr. Crocco GaléAs, G. - IACOBINI, C.: Lo sviluppo del tipo verbale parasintetico in latino: i prefissi ad-, in-, ex-. Quaderni Pataviani di Linguistica 12 (1993) 31-68 e IACOBINI, C.: Prefissazione; Parasintesi. In GRossmann, M. - RAINER, F. (a cura di): La formazione delle parole in italiano. Tübingen 2004, 99-163; 167-188, here 171. 